\title{
Utilidad de los parches para el análisis de drogas en sudor en un grupo de reclusos de Madrid
}

\author{
Milagros Vegue*; Enrique Álvaro**; Fabio Sternberg**; Mario Martín***; David Martínez**** \\ * Médico. Centro Penitenciario Madrid IV, Navalcarnero (Madrid) \\ * Médico. C.I.S. Victoria Kent, Madrid \\ *** Enfermero. C.I.S. Victoria Kent, Madrid \\ **** Médico. Dpto. de Medicina Preventiva y Salud Pública. Facultad de Medicina, Universidad Complutense, Madrid \\ Enviar correspondencia a: \\ Enrique Alvaro Brun. C.I.S. Victoria Kent, Servicios Sanitarios. C/ Juan de Vera 10, 28.045 MADRID. E-mail: med008045@saludalia.com
}

Recibido: 8 de Junio de 2004.

Aceptado: 7 de Febrero de 2005.

\section{RESUMEN}

Objetivo: Conocer la utilidad y la aceptación de los sistemas recolectores de sudor para la determinación de drogas en población penitenciaria. Comparar los resultados de los análisis de muestras de sudor y orina.

Material y método: Estudio descriptivo de tipo trasversal en una muestra de 51 personas en situación de Tercer Grado penitenciario o libertad condicional en las que se analizó el consumo de opiáceos y cocaína en parches y muestras de orina.

Resultados: El 82,35\% de la muestra consideraba que los sistemas recolectores de sudor son un buen procedimiento para determinar el consumo de drogas. El 81,65\% de los parches se encontraban totalmente adheridos a la piel al retirarlos, mientras que el $18,35 \%$ estaban parcialmente o totalmente despegados. De los 170 parches analizados en el 14,12\% se detectó cocaína, en el 0,59\% opiáceos y en el 2,94\% las 2 sustancias, encontrando asociación entre estos resultados y los de los análisis de orina $(p<0,0001)$.

Discusión: Resulta sencillo aplicar los parches y cuando se despegan se descubre fácilmente. Es posible que los parches despegados se desprendan espontáneamente como consecuencia de la sudoración abundante que aparece en sujetos que reciben altas dosis de metadona o que se produce por las altas temperaturas del verano. Aunque tampoco es posible excluir que los parches hayan sido voluntariamente desprendidos ya que su estado se asocia con la mala aceptación previa del procedimiento.

Palabras clave: Cárcel, presos, análisis de drogas, sudor, orina.

\section{ABSTRACT}

Objective: Ascertaining the usefulness and acceptance of sweat collection systems in order to establish the use of drugs in prison populations. Comparing the results of sweat and urine sample analyses.

Methodology: Observational cross-sectional study in a sample of 51 Grade-Three inmates or on parole involving an analysis of opiate and cocaine use on the basis of patches and urine samples.

Results: $82.35 \%$ of the sample considered that sweat collection systems are a good procedure in determining drug use. $81.75 \%$ of the patches were found completely adhered to the skin at removal, whereas $22.7 \%$ were partially or completely unstuck. Of the 170 patches which were analysed, cocaine was detected in $14.12 \%$ of them, opiates in $0.59 \%$, and both substances were detected in $2.94 \%$ patches. An association was found when comparing these results with those from the urine analyses ( $p<0.0001)$.

Discussion: Patches are easy to apply and, when they detach easy to detect. It is possible that unstuck patches have become spontaneously detached as a result of the excessive perspiration found in patients receiving high doses of methadone or from high summer temperatures. However, the possibility that the patches have been voluntarily detached cannot be excluded.

Key words: Prison, inmates, drug analysis, sweat, urine.

\section{INTRODUCCIÓN}

E toxicología clínica actualmente se diferencian dos áreas de trabajo, la médica y la legal11. Por un lado se realizan análisis con finalidad médica en el medio hospitalario o para el seguimiento de pacientes incluidos en programas de tratamiento. Pero además se demandan este tipo de pruebas en el ámbito laboral2 ${ }^{2}$ de la seguridad del trafico ${ }^{3}$, judicial o penitenciario, en este caso para la clasificación interior, concesión de permisos de salida, libertad condicional y beneficios penitenciarios ${ }^{4,5}$.

Los análisis con fines legales se diferencian de los realizados con finalidad médica en que pueden ocasionar consecuencias negativas para los intereses del individuo, y por tanto es necesario que sus resultados 
sean legalmente defendibles ${ }^{6}$. Esto es, es preciso que alcancen certeza legal, que permite razonablemente fijar una conclusión que responda a criterios científicos y lógicos, aún sin lograr la evidencia absoluta.

Desde hace años se realizan determinaciones de drogas en sudor $7,8,9$, que puede recogerse con un algodón cosmético de la superficie de la piel, cuando interesa detectar consumos recientes ${ }^{10,11}$, o mediante la aplicación de parches epidérmicos formados por una almohadilla de absorción que al estar en contacto con la piel acumula el sudor, y que se sujeta por una cubierta protectora que se pega por medio de un adhesivo hipoalérgico. Esta membrana permite el paso de vapor de agua, oxígeno y dióxido de carbono, lo que facilita su utilización durante períodos de al menos 7 días, pero no permite el paso de moléculas de mayor tamaño de forma que las drogas quedan atrapadas en la almohadilla ${ }^{12}$. Para acortar el tiempo mínimo de uso se ha diseñado un dispositivo calefactor que puede colocarse encima del parche normal para favorecer la transpiración ${ }^{13}$.

Los parches recolectores tienen la ventaja de que en el sudor las drogas se metabolizan en menor medida que en la orina, pero sobre todo con ellos se ensancha notablemente la ventana de detección, permitiendo la supervisión continúa, por lo que se previene el uso de drogas al disuadir al usuario del consumo ${ }^{14}$.

La interpretación de los resultados de los análisis debe ser cualitativa, como sucede con la orina, ya que la cantidad de sudoración se influye por las diferencias individuales y además se condiciona por la actividad física del sujeto y por la temperatura ambiente ${ }^{15,16}$. Por tanto los resultados sólo indican la presencia o ausencia de droga medida por encima o por debajo del punto de corte de la técnica analítica sin que se puedan realizar interpretaciones fidedignas sobre la dosis administrada y el tiempo trascurrido desde que se produjo la toma.

Por otro lado hay que considerar la capacidad de detección que tiene esta muestra, pues como el sudor resulta menos sensible para la detección de cocaína y opiáceos que el uroanálisis tres veces por semana pueden aparecer falsos negativos ${ }^{14,17}$. Aunque la posibilidad de que aparezcan falsos positivos tiene más importancia cuando la prueba puede tener implicaciones legales. Estos falsos positivos han sido atribuidos a la contaminación de las muestras por dos procedimientos. En primer lugar porque en la piel, a semejanza de lo que sucede en el pelo, pueden permanecer las drogas a pesar de haber seguido las instrucciones recomendadas para la limpieza cutánea previa a la aplicación del parche. En segundo lugar porque es posible que se produzca contaminación externa, ambiental, ya que se ha demostrado que el parche no es totalmente impermeable al paso de dro$\operatorname{gas}^{18,19,20,21}$.

\section{MATERIAL Y MÉTODO}

\section{Objetivo}

Conocer la utilidad de las muestras de sudor para la determinación de drogas en población penitenciaria. Determinar el nivel de aceptación de los parches recolectores de sudor en reclusos. Comparar los resultados de los análisis de muestras de sudor y orina.

\section{Muestra}

Participaron en el estudio sujetos vinculados al Centro de Inserción Social Victoria Kent de Madrid que cumplían condena en Tercer Grado penitenciario o se encontraban en situación de libertad condicional en tratamiento con metadona. El Tercer Grado penitenciario permite al interno desarrollar distintas actividades en el exterior durante el día y pernoctar en la cárcel. La libertad condicional se aplica a los penados que, cumpliendo los requisitos que señala el Código Penal, pasan en libertad el último período de la condena con la obligación de someterse al control de los Servicios Sociales Penitenciarios y observar determinadas reglas de conducta como puede ser el realizar tratamiento de su drogodependencia.

La selección de las muestras se realizó de de forma correlativa a medida que ingresaban en el centro internos en Tercer Grado con o sin antecedentes de drogodependencia, o iniciaban tratamiento con metadona en este recurso los liberados condicionales, además se incluyeron aquellos pacientes que encontrándose anteriormente en tratamiento con metadona presentaban resultados positivos en los controles de orina.

Se facilitó información suficiente sobre el estudio y seguidamente se solicitó la colaboración voluntaria de los participantes. Se aplicaron los siguientes criterios de exclusión: negativa a colaborar, previsible abandono del centro o permiso penitenciario en el mes siguiente, presencia de lesiones cutáneas que contraindicaban la aplicación y razones de fuerza mayor que impedían la asistencia semanal a consulta. Sólo 2 internos de los seleccionados no aceptaron colaborar.

De los 54 sujetos que aceptaron colaborar en el trabajo 3 liberados condicionales (5,55\%) no fueron incluidos ya que abandonaron antes de la retirada del primer parche. La muestra de 51 sujetos esta formada por 43 hombres $(84,31 \%)$ y 8 mujeres $(15,69 \%)$. La edad media es de 36,45 años (rango: 21-55 años y desviación típica 7,22). Se encuentran en Tercer 
Grado penitenciario 18 sujetos $(35,29 \%)$ y en libertad condicional $33(64,71 \%)$. Reciben tratamiento con metadona los 33 liberados condicionales y 5 de los participantes que cumplen condena en Tercer Grado penitenciario, en total 38 personas $(74,51 \%)$, con dosis media de 83,92 mg/día (rango: 14-230 y desviación típica 52,31).

\section{Procedimiento}

Se trata de un estudio descriptivo de tipo trasversal, realizado entre mayo de 2003 y abril de 2004, en el que se investiga el consumo de drogas en la muestra de población penitenciaria seleccionada mediante la colocación de forma sucesiva por personal de enfer-

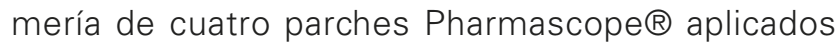
durante una semana, y la recogida de muestras de orina coincidiendo con los días de colocación, cambio y retirada de parches.

Los parches se colocan en zonas sin vello, no íntimas y cubiertas por la ropa, entre aquellos lugares recomendados en las instrucciones de uso y atendiendo a las sugerencias de los usuarios.

A cada participante se le asignó un código de identificación y de cada uno se recogieron datos básicos como la edad, sexo, clasificación penitenciaria, tratamiento con metadona, dosis que recibe y valoración que realiza de la determinación de drogas de abuso en sudor previo a la colocación del primer parche. Además para cada parche colocado se registró el lugar de aplicación, la fecha de colocación y retirada, la aparición de reacciones adversas, el estado del parche en el momento de la retirada, y el resultado del análisis realizado. También se registraron las fechas de emisión y los resultados del análisis de las muestras de orina.

En cada parche se analizaron opiáceos, 6monoacetilmorfina y cocaína mediante enzimoinmunoensayo, inmunofluorescencia o fluorescencia polarizada mediante el autoanalizador Cobas MIRA S de Roche Diagnostic y el analizador de fluorescencia TDX de Abbott. En orina se analizó cocaína y opiáceos mediante inmunoensayo con el analizador Viva Vitalab y el QuikPacll OneStep.

En el análisis estadístico se utilizaron las pruebas de $\chi^{2}$ (Chi cuadrado) y $t$ (t de Student), con nivel de confianza del 95\% mediante el Programa de Análisis Estadístico G-Stat.

\section{RESULTADOS}

De los 51 participantes incluidos en el estudio, 35 $(68,63 \%)$ completaron el protocolo recibiendo 4 par- ches, mientras que $16(31,37 \%)$ no lo completaron, 1 interno en Tercer Grado (1,96\%) al ser trasladado a otro centro penitenciario, y 15 liberados condicionales $(29,41 \%)$. Entre estos, al alcanzar la libertad definitiva 2 (3,92\%), por motivos de trabajo 2 (3,92\%), al derivarlo a otro centro atención a drogodependientes 1 $(1,96 \%)$ y al abandonar 10 (19,61\%), aduciendo que presentaban reacción local, caída del parche por hipersudoración o imposibilidad para acudir al centro.

Antes de la aplicación del primer parche consideraban que estas pruebas son un buen procedimiento para la determinación de drogas 42 participantes $(82,35 \%)$, los aceptaban parcialmente $7(13,73 \%)$, y 2 $(3,92 \%)$ señalaron que los parches son un mal procedimiento para detectar drogas.

Se colocaron 173 parches en los 51 sujetos, se retiraron 170, pues 3 internos se deshicieron de los parches antes de poder retirarlos. Se localizaron 28 en la región braquial $(16,47 \%), 62$ en la escapular $(36,47 \%), 57$ en la deltoidea $(33,53 \%)$ y 23 en la lumbar $(13,53 \%)$. El tiempo medio de aplicación fue de 7,27 días, en dos ocasiones un parche tuvo que sustituirse a las 24 horas ya que el participante acudió a consulta señalando que el parche se le había desprendido en ese plazo, en cambio 2 parches se mantuvieron 14 días y al retirarlos se encontraban en buen estado.

Se analizaron 170 parches, en 140 (82,35\%) no se encontraron opiáceos o cocaína, y se detectaron drogas en $30(17,65 \%)$, opiáceos en 1 (0,59\%), cocaína en $24(14,12 \%)$, y las dos sustancias en $5(2,94 \%)$. Se recogieron 209 muestras de orina, la mayoría sin supervisión directa, de las que 181 (86,6\%) resultaron negativas a opiáceos y cocaína. En las 28 (13,4\%) muestras en que se detectaron drogas se encontraron $9(4,3 \%)$ resultados positivos a opiáceos y cocaína, 1 $(0,5 \%)$ a opiáceos y $18(8,6 \%)$ a cocaína.

En el momento de ser retirados 129 parches $(81,65 \%)$ se encontraban totalmente adheridos a la piel, $14(8,86 \%)$ estaban parcialmente despegados y $15(9,49 \%)$ totalmente despegados. No se tomaron en consideración 12 parches al presentar signos o reconocer el usuario que habían sido manipulados.

Apareció reacción adversa local en el momento de la retirada, eritema, edema o sensación de quemazón en 17 parches, (10\%), mientras que en 153, (90\%), no apareció reacción de ningún tipo.

Comparando los resultados positivos a opiáceos o cocaína en parches versus orina recogida al colocar o retirar cada parche, se aprecia claramente la asociación entre los resultados de los análisis de los dos especímenes $\left(\chi^{2}=27,30, p<0,0001\right)$. Algunos consumos sólo se detectaron en los parches, ya sea por manipulación de la muestra de orina o por haberse realizado fuera de la ventana de detección de este 
espécimen. En otros casos los consumos se evidenciaron únicamente en el análisis de orina, y al encontrarse los parches completamente adheridos en el momento de ser retirados, estas diferencias pueden explicarse, como se señaló anteriormente, porque los sistemas recolectores de sudor resultan menos sensibles ya que en este fluido los niveles de droga son inferiores a los que se encuentran en la orina.

\section{DISCUSIÓN}

Los parches epidérmicos han resultado sencillos de utilizar ya que se aplican y se retiran con facilidad, no son necesarias medidas de refrigeración para su conservación, y al poder dividirse las almohadillas recolectoras en dos mitades se puede disponer fácilmente de muestras para contraanálisis.

Este procedimiento presenta otras ventajas en comparación con las determinaciones en orina. Es un dispositivo no invasor que, a diferencia de la orina con supervisión directa, no necesita instalaciones especiales, no afecta a la intimidad del individuo y no precisa de personal del mismo sexo para la recogida de muestras. Ha resultado difícil de manipular o falsificar ya que cuando el parche se ha despegado se aprecia fácilmente en la película plástica que lo sujeta, además en este caso no es posible diluir la muestra.

Los parches también tienen algunos inconvenientes, son caros, resultan menos sensibles que tres determinaciones de orina semanales y es posible que con ellos se presenten falsos positivos por contaminación medioambiental.

Los datos sobre consumo de drogas que se presentan no son de aplicación a la población penitenciaria general ya que la muestra estaba formada por un reducido número de sujetos que además eran principalmente drogodependientes en tratamiento con metadona.

Entre los resultados expuestos llama la atención que el $18,35 \%$ de los parches no manipulados se encontraban parcial o totalmente desprendidos en el momento de ser retirados. Cuando los parches se desprenden, aunque sea parcialmente, el resultado del análisis deja de ser fiable, no solo por la posibilidad de que aparezcan falsos negativos, sino también porque al contaminarse la almohadilla desde el exterior puede producirse un resultado falsamente positivo.

Es cierto que se detecta fácilmente el parche recolector que se ha despegado, pero surgen dudas ante la posibilidad de que se hayan desprendido espontáneamente, aunque no se encontró ninguna referencia a esta circunstancia en la bibliografía consultada.
Para identificar los factores que se asocian al desprendimiento del parche recolector de sudor se realizó un estudio de asociación estadística mediante el método del Chi cuadrado. En la tabla 1 se recogen estos resultados. Se encuentra asociación entre el estado del parche y cuatro variables, la estación del año, la dosis de metadona recibida, la aceptación previa y la edad de los usuarios.

La asociación entre el estado de los sistemas recolectores al retirarlos y la estación del año puede explicarse por la mayor sudoración que se produce cuando la temperatura es elevada, pudiendo ocasionar ese exceso de sudoración que el parche se desprenda espontáneamente.

No se observa asociación entre el estado del parche y el hecho de encontrarse en tratamiento sustitutivo a pesar de que la sudoración abundante es un efecto secundario persistente en las personas que toman metadona ${ }^{22}$. Pero cuando se considera el estado de los parches según la dosis de metadona recibida, los pacientes en tratamiento con metadona que presentaban parches totalmente adheridos recibieron dosis media de 73,74 mgr/día, mientras que los que los usuarios de metadona con parches parcial o totalmente despegados tomaban una dosis media de 101,46 mgr/día, encontrando diferencias estadísticamente significativas ( $t=-2,56 ; p=0,006)$. Esta diferencia de medias puede explicarse al considerar que en los pacientes con mayores dosis pueden aparecer más efectos secundarios, de forma que se favorezca el desprendimiento del parche por sudoración excesiva, pero también existen diferencias estadísticamente significativas entre la dosis de metadona recibida y la estación del año en que se coloca el parche $(t=-2$ y $p$ $=0,047)$, y entre esas dosis y la aceptación previa del procedimiento ( $t=-4,63$ y $p<0,0001)$.

Para estudiar como afecta la disposición previa del sujeto al estado del parche según la estación en que se utiliza, se estratificaron los sujetos según la aceptación. En los individuos con buena aceptación las diferencias en el estado del parche según la estación del año resultan significativas $\left(\chi^{2}=22,86, p<0,0001\right)$. En los sujetos que no refirieron buena aceptación previa las frecuencias absolutas en algunas celdas son muy bajas, por lo que al no resultar adecuada la prueba de $\chi^{2}$ se aplica la prueba exacta de Fisher, encontrando también diferencias estadísticamente significativas a un valor de $p=0,03$. En cambio, al estratificar los sujetos según la estación del año en que se coloca el parche no existen diferencias según la aceptación previa en el estado del dispositivo recolector.

En conclusión, los parches para la determinación de drogas en sudor son bien aceptados y han resultado útiles para controlar el uso de drogas en la muestra de población penitenciaria estudiada. Conviene mejorar su diseño a fin de evitar el desprendimiento 
Tabla 1. Comparación de las variables de estudio con el estado correcto vs. incorrecto del parche

\begin{tabular}{|c|c|c|c|c|c|c|}
\hline \multirow[b]{2}{*}{$\begin{array}{l}\text { Edad Media } \\
\text { en años (D.S.) }\end{array}$} & \multicolumn{2}{|c|}{ Correcto } & \multicolumn{2}{|c|}{ Incorrecto } & \multirow{2}{*}{$\begin{array}{c}\text { Prueba de Contraste } \\
\qquad t=-2,63\end{array}$} & \multirow{2}{*}{$\begin{array}{c}\mathbf{p} \\
0,0047^{*}\end{array}$} \\
\hline & 35,3 & $(5,95)$ & 38,7 & $(9,25)$ & & \\
\hline Edad Media & 73,7 & $(43,58)$ & 101,46 & (59.88) & $t=-2,56$ & $0,0059 *$ \\
\hline & $\mathbf{n}$ & $(\%)$ & $\mathbf{n}$ & $(\%)$ & & \\
\hline $\begin{array}{l}\text { Sexo } \\
\text { Hombre } \\
\text { Mujer }\end{array}$ & $\begin{array}{l}106 \\
24\end{array}$ & $\begin{array}{l}(81,54 \%) \\
(72,73 \%)\end{array}$ & $\begin{array}{c}24 \\
9\end{array}$ & $\begin{array}{l}(18,46 \%) \\
(27,27 \%)\end{array}$ & $\chi^{2}=1,2655$ & 0,2606 \\
\hline $\begin{array}{l}\text { Clasificación } \\
3^{\circ} \text { Grado } \\
\text { L. Condicional }\end{array}$ & $\begin{array}{l}56 \\
74\end{array}$ & $\begin{array}{l}(83,58 \%) \\
(77,08 \%)\end{array}$ & $\begin{array}{l}11 \\
22\end{array}$ & $\begin{array}{l}(16,42 \%) \\
(22,92 \%)\end{array}$ & $\chi^{2}=1,0321$ & 0,3097 \\
\hline $\begin{array}{l}\text { TMM } \\
\text { Si } \\
\text { No }\end{array}$ & $\begin{array}{l}92 \\
38\end{array}$ & $\begin{array}{l}(79,31 \%) \\
(80,85 \%)\end{array}$ & $\begin{array}{c}24 \\
9\end{array}$ & $\begin{array}{l}(20,69 \%) \\
(19,15 \%)\end{array}$ & $\chi^{2}=0,0492$ & 0,8245 \\
\hline $\begin{array}{l}\text { Estación } \\
\text { Verano } \\
\text { Resto del año }\end{array}$ & $\begin{array}{c}11 \\
119\end{array}$ & $\begin{array}{l}(39,29 \%) \\
(88,15 \%)\end{array}$ & $\begin{array}{l}17 \\
16\end{array}$ & $\begin{array}{l}(60,71 \%) \\
(11,85 \%)\end{array}$ & $\chi^{2}=34,2904$ & $<0,0001^{*}$ \\
\hline $\begin{array}{l}\text { Localización } \\
\text { Braquial } \\
\text { Escapular } \\
\text { Deltoidea } \\
\text { Lumbar }\end{array}$ & $\begin{array}{l}19 \\
53 \\
42 \\
16\end{array}$ & $\begin{array}{l}(76,00 \%) \\
(85,48 \%) \\
(82,35 \%) \\
(64,00 \%)\end{array}$ & $\begin{array}{l}6 \\
9 \\
9 \\
9\end{array}$ & $\begin{array}{l}(24,00 \%) \\
(14,52 \%) \\
(17,65 \%) \\
(36,00 \%)\end{array}$ & $\chi^{2}=5,5349$ & 0,1366 \\
\hline $\begin{array}{l}\text { Aceptación previa } \\
\text { Buena } \\
\text { Regular-Mala }\end{array}$ & $\begin{array}{c}118 \\
12\end{array}$ & $\begin{array}{l}(82,52 \%) \\
(60,00 \%)\end{array}$ & $\begin{array}{c}25 \\
8\end{array}$ & $\begin{array}{l}(17,48 \%) \\
(40,00 \%)\end{array}$ & $\chi^{2}=5,5098$ & $0,0189^{*}$ \\
\hline $\begin{array}{l}\text { Reacción adversa } \\
\text { Si } \\
\text { No }\end{array}$ & $\begin{array}{c}8 \\
122\end{array}$ & $\begin{array}{l}(66,67 \%) \\
(80,79 \%)\end{array}$ & $\begin{array}{c}4 \\
29\end{array}$ & $\begin{array}{l}(33,33 \%) \\
(19,21 \%)\end{array}$ & $\chi^{2}=1,3742$ & 0,2411 \\
\hline $\begin{array}{l}\text { Resultado parche } \\
\text { Negativo } \\
\text { Positivo }\end{array}$ & $\begin{array}{l}109 \\
21\end{array}$ & $\begin{array}{l}(81,95 \%) \\
(70,00 \%)\end{array}$ & $\begin{array}{c}24 \\
9\end{array}$ & $\begin{array}{l}(18,05 \%) \\
(30,00 \%)\end{array}$ & $\chi^{2}=2,1667$ & 0,1410 \\
\hline $\begin{array}{l}\text { Resultado orina } \\
\text { Negativo } \\
\text { Positivo }\end{array}$ & $\begin{array}{c}110 \\
18\end{array}$ & $\begin{array}{l}(82,71 \%) \\
(75,00 \%)\end{array}$ & $\begin{array}{c}23 \\
6\end{array}$ & $\begin{array}{l}(17,29 \%) \\
(25,00 \%)\end{array}$ & $\chi^{2}=0,8019$ & 0,3705 \\
\hline
\end{tabular}

espontáneo en pacientes que sudan abundantemente. Parece adecuado estudiar su aplicación en otras muestras de drogodependientes con problemas legales, como son los casos de suspensión o sustitución de penas y los permisos penitenciarios de reclusos en Segundo Grado.

\section{AGRADECIMIENTO}

A la Dirección, Servicios Sanitarios y Equipo Técnico del C.I.S. Victoria Kent de Madrid por su colaboración en la realización de este trabajo. 


\section{REFERENCIAS.}

1. Lora-Tamayo C, Tena T. Elementos para la interpretación médico-legal de los resultados de análisis toxicológicos de drogas de abuso. En: Delgado S, Torrecilla JM, directores. Medicina Legal en Drogodependencias. Madrid: Agencia Antidroga; 2001. p. 321-46.

2. Caplan YH, Goldberger BA. Alternative specimens for workplace drug testing. J Anal Toxicol. 2001; 25:396-9.

3. Kintz $P$, Cirimele V, Ludes B. Detection of cannabis in oral fluid (saliva) and forehead wipes (sweat) from impaired drivers. J Anal Toxicol. 2000 Oct;24(7):557-61.

4. Álvaro E, Vegue M, Spottorno I. Utilidad del análisis de drogas en orina en un centro penitenciario femenino. Adicciones 1993; 5: 257-73.

5. Wish E. Identificación de los delincuentes drogadictos. En: National Institute of Drug Abuse. Tratamiento obligatorio de la drogadicción, investigación y práctica clínica. Madrid: Fundación de Ayuda contra la Drogadicción; 1995: p. 139-56 [original 1988].

6. Doadrio J. Análisis de drogas de abuso. Programas de detección. En: Martínez M, Rubio G, directores. Manual de drogodependencias para enfermería. Madrid: Ediciones Díaz de Santos; 2002. p 327-38.

7. Dolan K, Rouen D, Kimber J. An overview of the use of urine, hair, sweat and saliva to detect drug use. Drug Alcohol Rev. 2004; 23:213-7.

8. Kidwell DA, Kidwell JD, Shinohara F, Harper C, Roarty K, Bernadt K, McCaulley RA, Smith FP. Comparison of daily urine, sweat, and skin swabs among cocaine users. Forensic Sci Int. 2003; 23:63-78.

9. Winhusen TM, Somoza EC, Singal B, Kim S, Horn PS, Rotrosen J. Measuring outcome in cocaine clinical trials: a comparison of sweat patches with urine toxicology and participant self-report. Addiction. 2003; 98:317-24.

10. Pacifici R, Farre M, Pichini S, Ortuno J, Roset PN, Zuccaro P, Segura J, de la Torre R. Sweat testing of MDMA with the Drugwipe analytical device: a controlled study with two volunteers. J Anal Toxicol. 2001; 25:144-6.

11. Samyn N, van Haeren C. On-site testing of saliva and sweat with Drugwipe and determination of concentra- tions of drugs of abuse in saliva, plasma and urine of suspected users. Int J Legal Med. 2000; 113:150-4.

12. PharmChek Drugs of Abuse Patch. The Continuous Monitoring Patch. Disponible en: http://www.csstest. com [consultado 17.12.04]

13. Liberty HJ, Johnson BD, Fortner N, Randolph D. Detecting crack and other cocaine use with fastpatches. Addict Biol. 2003; 8:191-200.

14. Huestis MA, Cone EJ, Wong CJ, Umbricht A, Preston $\mathrm{KL}$. Monitoring opiate use in substance abuse treatment patients with sweat and urine drug testing. J Anal Toxicol 2000; 24: 509-21.

15. Identification, Demonstration, and Assessment of NonInvasive Drug Screening Technology. Disponible en: http:/www.nlectc.org/nlectcse/download/blackburn_ feb2003_drugscreening.pdf/ [consultado 17.12.04].

16. Kintz P, Tracqui A, Marzullo C, Darreye A, Tremeau F, Greth $\mathrm{P}$, Ludes B. Enantioselective analysis of methadone in sweat as monitored by liquid chromatography/ ion spray-mass spectrometry. Ther Drug Monit. 1998; 20:35-40.

17. Crouch DJ, Cook RF, Trudeau JV, Dove DC, Robinson JJ, Webster $\mathrm{HL}$ et al. The detection of drugs of abuse in liquid perspiration. J Anal Toxicol 2001; 25: 625-27.

18. Improving the PharmcheckTM Sweat Patch: Reducing False Positives from Environmental Contamination and Increasing Drug Detection. Disponible en: http://www. ncjrs.org/pdffiles1/nij/196030.pdf [consultado 17.12.04]

19. Kidwell DA, Smith FP. Susceptibility of PharmChek drugs of abuse patch to environmental contamination. Forensic Sci Int. 2001;116: 89-106.

20. Levisky JA, Bowerman DL, Jenkins WW, Karch SB. Drug deposition in adipose tissue and skin: evidence for an alternative source of positive sweat patch tests. Forensic Sci Int. 2000;110:35-46.

21. Susceptibility of PharmChek ${ }^{\mathrm{TM}}$ Drugs of Abuse Patch to Environmental Contamination. Disponible en: http:// www.ncjrs.org/pdffiles1/nij/195986.pdf [consultado 17.12.04]

22. Duro P, Casas M. Programas de mantenimiento con agonistas opiáceos. En: Cadafalch J, Casas M. El paciente heroinómano en el Hospital General. Madrid: Delegación del Gobierno del Plan Nacional sobre Drogas; 1993. p. 105-29. 